\title{
Effects of Average Index Variation in Apodized Long-Period Fiber Gratings
}

\author{
Yanju GU and Kin Seng CHIANG* \\ Department of Electronic Engineering, City University of Hong Kong, Hong Kong SAR, China \\ ${ }^{*}$ Corresponding author: Kin Seng CHIANG_Ｅ-mail: eeksc@cityu.edu.hk
}

\begin{abstract}
We analyze the effects of average index variation on the transmission characteristics of an index-apodized long-period fiber grating (LPFG) by the transfer matrix method and study how these effects depend on the grating length, the grating profile, the modal dispersion factor, and the duty cycle of the index modulation. Apart from shifting the resonance wavelength and modifying the rejection band, average index variation can give rise to significant side lobes that may appear on the short-wavelength or long-wavelength side of the rejection band, depending on the signs of the average index change and the modal dispersion factor. Our results provide general guidance for the writing of LPFGs for the minimization of side lobes. Our analysis compares well with published experimental results and should be useful for the design and fabrication of LPFGs.
\end{abstract}

Keywords: Apodization, fiber grating, long-period fiber grating (LPFG), optical fiber sensor, optical filter, transfer matrix method

Citation: Yanju GU and Kin Seng CHIANG, "Effects of Average Index Variation in Apodized Long-Period Fiber Gratings," Photonic Sensors, vol. 3, no. 2, pp. 102-111, 2013.

\section{Introduction}

Long-period fiber grating (LPFG) [1], typically formed by periodically modulating the refractive index along a single-mode fiber, enables the guided core mode to couple to the cladding modes at specific wavelengths. Being simple band-rejection filters, LPFGs have found many applications in optical communication [2], signal processing [3], and sensing [4]. The cladding modes of the fiber are sensitive to the surrounding medium, which makes LPFGs particularly attractive for sensor applications. The transmission spectrum of a uniform LPFG exhibits side lobes around the rejection band, which are undesirable in certain applications [5]. In principle, it is possible to completely eliminate side lobes by using an apodized LPFG, where the index modulation is gradually reduced to zero towards the two ends of the grating [6]. In practice, the magnitude [7] and/or the duty cycle [8] of the index modulation can be used to control the degree of apodization. However, the index change along such an apodized grating is usually of the same sign and thus gives rise to an average index that varies slowly along the grating. In the case of an apodized fiber Bragg grating (FBG), a slowly varying background index can significantly broaden the reflection peak and generate large side lobes on one side of the reflection peak [9] in as much the same way as chirping the grating period [10]. The average index effects in an FBG can be understood as the results of Fabry-Perot resonances produced by the two edges

Received: 14 June 2012 / Revised version: 9 January 2013

(C) The Author(s) 2013. This article is published with open access at Springerlink.com 
of the grating [11]. Similar phenomena have been observed experimentally with an apodized LPFG that has a varying background index, which are interpreted as the interference effects in a Mach-Zehnder interferometer [8]. A detailed analysis of ideal apodized LPFGs that ignores the average index variation is available [6]. The present study extends the previous work by including the effects of average index variation that exists in practical apodized LPFGs.

Our analysis is based on the transfer matrix method [6], where a non-uniform grating is divided into a number of concatenated uniform grating sections, and the coupling strength and the resonance wavelength are allowed to vary from section to section. We also incorporate the waveguide dispersion properties of the coupling modes into the analysis. We discuss, in particular, the effects of average index variation on the rejection band and the side lobes, and how the effects change with the grating length, the apodization profile, and the order of the cladding mode. We investigate the extent to which the side lobes can be reduced by control of the grating profile and the duty cycle of the index modulation. Finally, we compare our simulation results with published experimental data. Our study should be useful for the design and fabrication of apodized LPFGs.

\section{Method of analysis}

We consider an LPFG with the following index variation along the core of a single-mode fiber:

$$
\Delta n(z)=\Delta n_{\mathrm{dc}}(z)+\Delta n_{\mathrm{ac}}(z) \sin \left(\frac{2 \pi}{\Lambda} z+\varphi\right)
$$

where $z$ is the distance along the fiber $(0 \leq z \leq L), \Lambda$ is the grating period, $L$ is the grating length, $\Delta n_{\mathrm{dc}}(z)$ is the background index variation, $\Delta n_{\mathrm{ac}}(z)$ is the grating amplitude variation, and $\varphi$ is a constant phase. The "dc" component $\Delta n_{\mathrm{dc}}(z)$ can be positive or negative, while the "ac" amplitude $\Delta n_{\mathrm{ac}}(z)$ is taken to be positive.
As $\Delta n_{\mathrm{dc}}(z)$ and $\Delta n_{\mathrm{ac}}(z)$ are slowly varying functions of $z$, we can approximate the grating profile with $M(>1)$ uniform sections with lengths $z_{1}, z_{2}, \cdots, z_{M}$, as illustrated in Fig. 1. The dc and ac index changes in the $i$ th $(i=1,2, \cdots, M)$ section, denoted as $\Delta n_{\mathrm{dc} i}$ and $\Delta n_{\mathrm{ac} i}$, respectively, are the values of the corresponding functions $\Delta n_{\mathrm{dc}}(z)$ and $\Delta n_{\text {ac }}(z)$ evaluated at the mid point of the section. The initial phase for each section is $\varphi_{i+1}=\varphi_{i}+(2 \pi / \Lambda) z_{i}$ $(i=1,2, \cdots, M-1)$ with $\varphi_{1}=\varphi$.

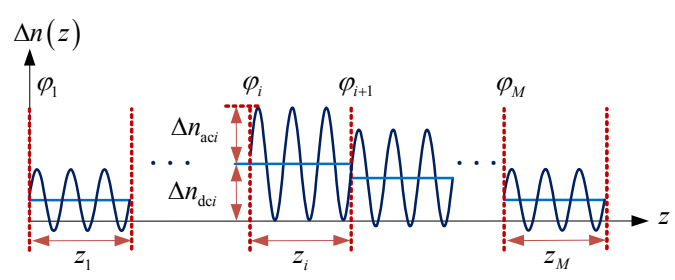

Fig. 1 Schematic diagram of a multi-section LPFG with a varying average index.

Over the spectral range of concern, the $\mathrm{LP}_{01}$ core mode couples only to one of the $\operatorname{LP}_{0 m}(m>1)$ cladding modes. We define a nominal resonance wavelength as

$$
\lambda_{0}=\left[N_{01}\left(\lambda_{0}\right)-N_{0 m}\left(\lambda_{0}\right)\right] \Lambda
$$

where $N_{01}$ and $N_{0 m}$ are, respectively, the effective indices of the $\mathrm{LP}_{01}$ and $\mathrm{LP}_{0 m}$ modes of the fiber in the absence of the grating. $\lambda_{0}$ is in fact the resonance wavelength of the ideal apodized LPFG that has no dc index change, i.e., $\Delta n_{\mathrm{dc}}(z)=0$. In the presence of average index variation, the effective indices in different sections are in general different, which results in different resonance wavelengths. The resonance wavelength in the $i$ th section is

$$
\lambda_{0 i}=\left[N_{01 i}\left(\lambda_{0 i}\right)-N_{0 m i}\left(\lambda_{0 i}\right)\right] \Lambda
$$

where $N_{01 i}$ and $N_{0 m i}$ are the corresponding effective indices. By treating the $\mathrm{dc}$ index change as a perturbation of the original fiber, we obtain [12]

$$
N_{0 p i}(\lambda)=N_{0 p}(\lambda)+\Delta n_{\mathrm{dci}} \eta_{0 p}, p=1 \text { or } m
$$

where $\lambda$ is the wavelength, $\eta_{0 p}$ is the confinement factor of the $\mathrm{LP}_{0 p}$ mode in the core area, which is assumed to be a constant in the spectral range of concern. To relate $\lambda_{0 i}$ to $\lambda_{0}$, we expand the effective 
indices at $\lambda_{0}$

$$
N_{0 p}(\lambda) \cong N_{0 p}\left(\lambda_{0}\right)+\frac{d N_{0 p}}{d \lambda}\left(\lambda-\lambda_{0}\right)
$$

and substitute the result into (4) and then into (3). With the help of (2), we find

$$
\lambda_{0 i}=\lambda_{0}+\gamma \Delta n_{\mathrm{dci}}\left(\eta_{01}-\eta_{0 m}\right) \Lambda
$$

where

$$
\gamma=\left[1-\Lambda\left(\frac{d N_{01}}{d \lambda}-\frac{d N_{0 m}}{d \lambda}\right)\right]^{-1}
$$

is the modal dispersion factor evaluated at $\lambda_{0}$ [13]. In general, $\eta_{01}$ is much larger than $\eta_{0 m}$. Whether $\lambda_{0 i}$ is larger or smaller than $\lambda_{0}$ depends on the sign of the dc index change $\Delta n_{\mathrm{dc} i}$ and the sign of the modal dispersion factor $\gamma$.

According to the transfer matrix method $[6,14$, 15], each uniform section is represented by a $2 \times 2$ matrix, and the output fields of the modes are obtained from the input fields by multiplying the characteristic matrices of all the uniform sections. With the electric fields of the $\mathrm{LP}_{01}$ and $\mathrm{LP}_{0 m}$ modes along the grating denoted as $E_{01}(z)$ and $E_{0 m}(z)$, respectively, the result is expressed as

$$
\left[\begin{array}{c}
E_{01}(L) \\
E_{0 m}(L)
\end{array}\right]=F_{M} \cdots F_{i} \cdots F_{2} F_{1}\left[\begin{array}{c}
E_{01}(0) \\
E_{0 m}(0)
\end{array}\right]
$$

with

$$
\begin{aligned}
F_{i}= & {\left[\begin{array}{cc}
e^{-j\left(\beta_{01 i}-\delta_{i}\right) z_{i}} & 0 \\
0 & e^{-j\left(\beta_{0 m i}+\delta_{i}\right) z_{i}}
\end{array}\right] . } \\
& {\left[\begin{array}{cc}
\cos q_{i} z_{i}-j \frac{\delta_{i}}{q_{i}} \sin q_{i} z_{i} & \frac{\kappa_{i}}{q_{i}} e^{-j \varphi_{i}} \sin q_{i} z_{i} \\
-\frac{\kappa_{i}}{q_{i}} e^{j \varphi_{i}} \sin q_{i} z_{i} & \cos q_{i} z_{i}+j \frac{\delta_{i}}{q_{i}} \sin q_{i} z_{i}
\end{array}\right] }
\end{aligned}
$$

In the above expression, $\beta_{01 i}=(2 \pi / \lambda) N_{01 i}$ and $\beta_{0 m i}=(2 \pi / \lambda) N_{0 m i}$ are the propagation constants of the $\mathrm{LP}_{01}$ and $\mathrm{LP}_{0 m}$ modes, respectively, $q_{i}=\left(\delta_{i}^{2}+\kappa_{i}^{2}\right)^{1 / 2}$, $\delta_{i}$ is the phase mismatch

$$
\begin{aligned}
\delta_{i} & \equiv \frac{1}{2}\left(\beta_{01 i}-\beta_{0 m i}\right)-\frac{\pi}{\Lambda} \\
& =\frac{\pi}{\gamma \Lambda}\left(\frac{\lambda_{0 i}}{\lambda}-1\right),
\end{aligned}
$$

and $\kappa_{i}$ is the coupling coefficient

$$
\kappa_{i}=\frac{\pi}{\lambda} I \Delta n_{\mathrm{ac} i}
$$

where $I$ is the overlap integral of the $\mathrm{LP}_{01}$ and $\mathrm{LP}_{0 m}$ mode fields over the core area. With the knowledge of the modal properties and the grating profile, the transmission spectrum of the grating $\left|E_{01}(L)\right|^{2} /\left|E_{01}(0)\right|^{2}$ can be readily calculated from (8).

Our formulation takes into account both the average index variation and the waveguide dispersion characteristics of the modes and thus enables a more realistic analysis of LPFGs.

\section{Effects of average index variation}

In this section, we analyze in detail the effects of average index variation on the transmission spectrum of an apodized LPFG with the transfer matrix method. Without losing generality, we assume that the dc and ac index changes follow the same profile, i.e., $\Delta n_{\mathrm{dc}}(z)=a_{0} \sigma(z)$ and $\Delta n_{\mathrm{ac}}(z)=$ $a_{1} \sigma(z)$, where $\sigma(z)$ is the normalized apodization profile, and $a_{0}$ and $a_{1}$ are the amplitudes of the corresponding index changes.

For the simulation work, we assume that the apodization function has the form $\sigma(z)=$ $\cos ^{N}[(\pi / L)(z-L / 2)]$, where $N$ is the profile-shape parameter. An example of the profile is shown in Fig. 2. The profile values in different sections of the piece-wise approximation are given by

$$
\sigma_{i}=\cos ^{N}\left(\frac{\pi(2 i-M-1)}{2 M}\right), i=1,2, \cdots, M \text {. }
$$

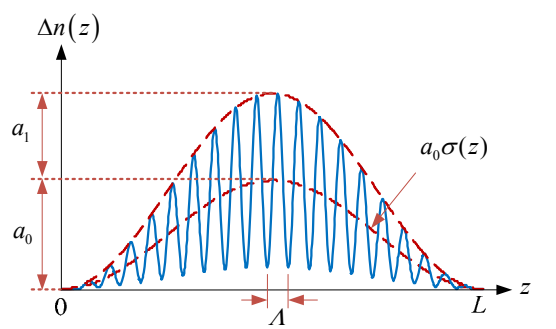

Fig. 2 A raised-cosine $(N=2)$ apodization profile showing the dc and ac components.

For the ideal apodized LPFG with a zero dc index change (i.e., $a_{0}=0$ ), the transmission at the resonance wavelength is given by [6] 


$$
\left|E_{01}(L)\right|^{2}=\left|E_{01}(0)\right|^{2} \cos ^{2}\left(\sum_{i=1}^{i=M} \kappa_{i} z_{i}\right) .
$$

When the value of the ac index change $a_{1}$ is set to satisfy the condition $\Sigma \kappa_{i} z_{i}=\pi / 2$, complete rejection is achieved for the LPFG with $a_{0}=0$ at the nominal resonance wavelength.

We consider a bare three-layer step-index fiber that has a core with an index of 1.45 and a radius of $3.63 \mu \mathrm{m}$ and a cladding with an index of 1.444 and a radius of $62.5 \mu \mathrm{m}$. The surrounding medium is air. Analytical formulae are available for the evaluation of the effective indices and the mode fields of the fiber [16]. We assume that light is launched only into the $\mathrm{LP}_{01}$ mode, i.e., $E_{01}(0) \neq 0$ and $E_{0 m}(0)=0$. Unless stated otherwise, ten uniform sections $(M=$ $10)$ are used in the simulation, and the grating period $\Lambda$ is fixed at $343 \mu \mathrm{m}$, which ensures coupling between the $\mathrm{LP}_{01}$ and $\mathrm{LP}_{07}$ modes at the nominal resonance wavelength $1.55 \mu \mathrm{m}$ (the value of $\gamma$ is 1.1).

\subsection{General effects}

Figure 3 shows the transmission spectra of a raised-cosine $(N=2)$ apodized LPFG with a length of $L=60 \mathrm{~mm}$ calculated for different values of the dc-to-ac ratio $a_{0} / a_{1}(0,0.5,1.0$, and 1.5$)$, where the value of $a_{1}$ is fixed to satisfy $\Sigma \kappa_{i} z_{i}=\pi / 2$ at the wavelength $1.55 \mu \mathrm{m}$. The ratio $a_{0} / a_{1}$ is a measure of the importance of the dc component in the grating profile. As shown in Fig. 3, without dc index change $\left(a_{0} / a_{1}=0\right)$, complete rejection occurs at $1.55 \mu \mathrm{m}$, and no side lobes are present, as expected for an ideal apodized LPFG [6]. With dc index change, the resonance wavelength shifts to a longer wavelength, and the rejection band is weakened and broadened. Most importantly, side lobes appear on the short-wavelength side of the rejection band. The side lobes on the short-wavelength side can be understood as the result of interference between the core mode that propagates through the grating and the core mode that is coupled out to the cladding at the input end of the grating and then coupled back to the core at the output end of the grating. The grating operates as a Mach-Zehnder interferometer with varying optical paths and field amplitudes across the bandwidth of the grating. Here, a positive $a_{0}$ is assumed; a negative $a_{0}$ would lead to a resonance wavelength shifting to a shorter wavelength and side lobes appearing on the long-wavelength side of the rejection band. As the value of $a_{0} / a_{1}$ increases, the rejection band becomes weaker and broader, and the side lobes become stronger, which can be explained by the larger spread of the resonance wavelength along the grating.

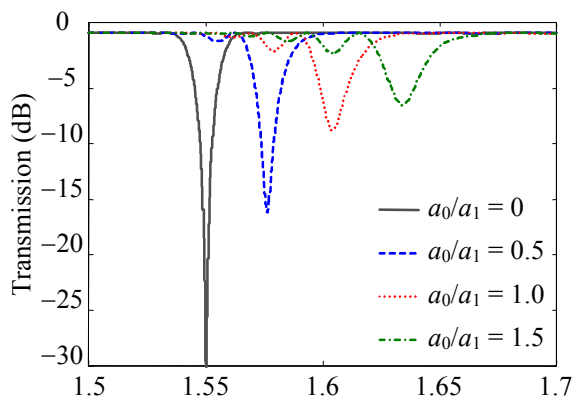

Fig. 3 Transmission spectra of a 60 -mm-length raised-cosine apodized LPFG calculated for four different values of $a_{0} / a_{1}$ : 0 , $0.5,1.0$, and 1.5 , where $a_{1}$ is fixed to ensure complete rejection at the nominal wavelength $1.55 \mu \mathrm{m}$.

\subsection{Grating length}

To demonstrate the effects of the grating length, we consider three raised-cosine $(N=2)$ apodized LPFGs that have different lengths: $L=40 \mathrm{~mm}$, $60 \mathrm{~mm}$, and $80 \mathrm{~mm}$. The ac index modulation profiles of the gratings are shown in Fig.4(a). The amplitudes of the ac index changes of the gratings are adjusted to ensure complete rejection at the nominal resonance wavelength (i.e., $\Sigma \kappa_{i} z_{i}=\pi / 2$ ). The variations of the transmission characteristics of the three gratings with the ratio $a_{0} / a_{1}$ are shown in Figs. 4(b)-4(e). As shown in Figs.4(b) and 4(d), the effects of the ratio $a_{0} / a_{1}$ on the resonance wavelength and the 3-dB bandwidth of the rejection band are stronger for a shorter grating, which can be explained by the larger range of the dc index change and hence the larger spread of the resonance wavelength in the shorter grating. Because the 
bandwidth is inversely proportional to the grating length, a shorter grating has a larger bandwidth [17]. On the other hand, as shown in Figs.4(c) and 4(e), the effects of the ratio $a_{0} / a_{1}$ on the strengths of the rejection band and the side lobe are practically independent of the grating length, which is consistent with the previous finding that the contrasts of the side lobes of a uniform LPFG with $\kappa L=\pi / 2$ do not depend on the grating length [6].

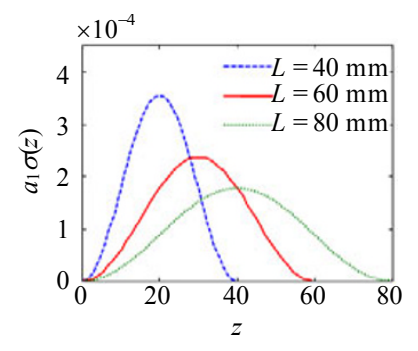

(a)

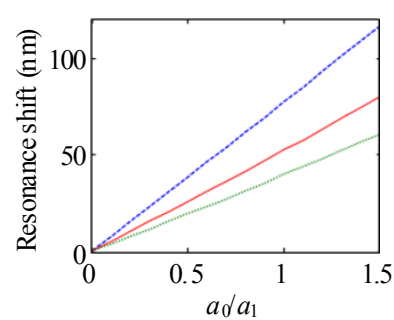

(b)

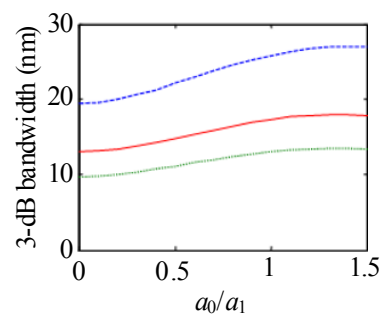

(d)

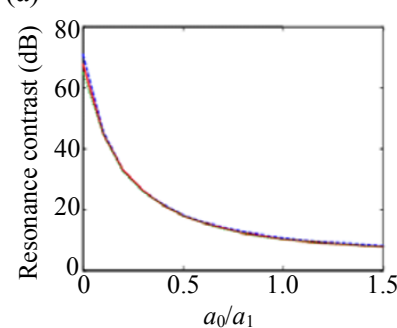

(c)

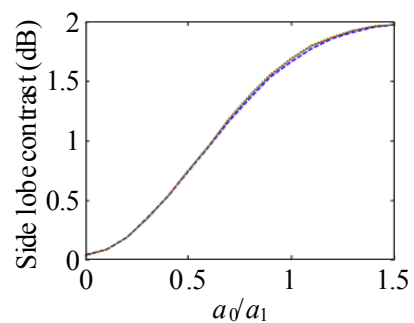

(e)
Fig. 4 Effects of the ratio $a_{0} / a_{1}$ on the transmission characteristics of (a) three raised-cosine apodized LPFGs with $L=40 \mathrm{~mm}, 60 \mathrm{~mm}$, and $80 \mathrm{~mm}$, (b) the resonance wavelength shift, (c) the contrast of the rejection band, (d) the 3-dB bandwidth of the rejection band, and (e) the contrast of the most significant side lobe.

\subsection{Apodization profile}

We consider four 60-mm-length apodized LPFGs that are characterized by different profile shapes: $N=0.5,1.0,1.5$, and 2.0, as shown in Fig. 5(a). Again, the amplitudes of the ac index changes of the gratings are adjusted to ensure complete rejection at the nominal resonance wavelength (i.e., $\left.\sum \kappa_{i} z_{i}=\pi / 2\right)$. As $N$ increases, the profile becomes narrower, and the maximum value of $a_{1}$ required for achieving $\sum \kappa_{i} z_{i}=\pi / 2$ becomes larger. The variations of the transmission characteristics of the gratings with the ratio $a_{0} / a_{1}$ are shown in Figs. 5(b)-5(e). The grating with a narrower profile (i.e., a larger value of $N$ ) has a shorter effective length and therefore shows a larger shift in the resonance wavelength and a broader 3-dB bandwidth. In general, the effects of the ratio $a_{0} / a_{1}$ on the resonance wavelength shift, the

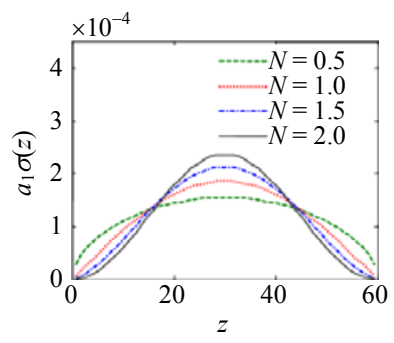

(a)

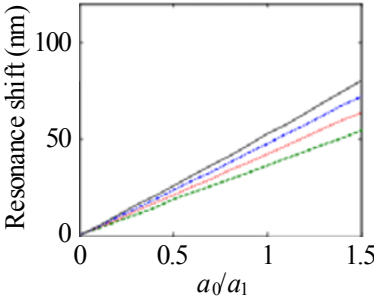

(b)

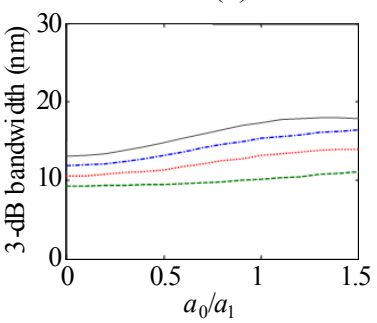

(d)

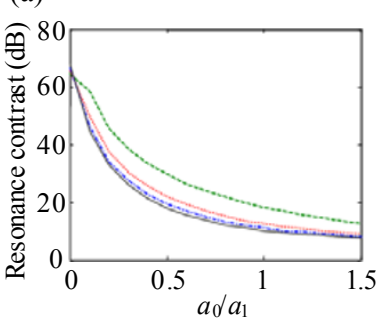

(c)

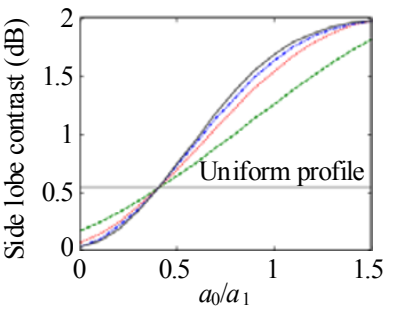

(e)
Fig. 5 Effects of the ratio $a_{0} / a_{1}$ on the transmission characteristics of (a) four 60-mm apodized LPFGs with $N=0.5$, $1.0,1.5$, and 2.0 , (b) the resonance wavelength shift, (c) the contrast of the rejection band, (d) the 3-dB bandwidth of the rejection band, and (e) the contrast of the most significant side lobe.

contrast of the rejection band, and the $3-\mathrm{dB}$ bandwidth are stronger for a narrower profile, as shown in Figs. 5(b)-5(d). The effects of the ratio 
$a_{0} / a_{1}$ on the contrast of the most significant side lobe are shown in Fig. 5(e), where the result for a uniform profile is also given. At $a_{0} / a_{1}<0.4$, a narrower profile produces a weaker side lobe, i.e., the $N=2$ profile is the most effective one for suppressing side lobes. On the other hand, at $a_{0} / a_{1}>0.4$, a narrower profile produces a stronger side lobe. We should note that a constant dc index change along a uniform grating does not affect the strengths of the side lobes; it only causes a shift in the resonance wavelength. As shown in Fig. 5(e), the side lobes generated by the dc index variation in an apodized grating can be much stronger than those of a uniform grating.

\subsection{Modal dispersion factor}

According to (6), the spread of the resonance wavelength in an apodized LPFG that has a dc index variation depends on the value of the modal dispersion factor $\gamma$. For a typical fiber, $\gamma$ is positive for low-order cladding modes and negative for high-order cladding modes, and its magnitude can be large for some modes in between [18]. The modal dispersion factors of the first twenty cladding modes of the fiber calculated at $1.55 \mu \mathrm{m}$ are shown in Fig. 6(a). For a positive dc index change, a positive (negative) $\gamma$ gives rise to a red (blue) shift in the resonance wavelength. In other words, a red (blue) shift in the resonance wavelength is expected for low-order (high-order) cladding modes. The situation is reversed for a negative dc index change.

Figure 6(b) shows the transmission spectra of four 60 -mm-length raised-cosine apodized LPFGs with $a_{0} / a_{1}=1.0$, which are designed for operation with the $\mathrm{LP}_{07}$ mode $(\Lambda=343 \mu \mathrm{m}, \gamma=1.1, I=0.109)$, the $\mathrm{LP}_{09}$ mode $(\Lambda=252 \mu \mathrm{m}, \gamma=2.7, I=0.118)$, the $\mathrm{LP}_{014}$ mode $(\Lambda=128 \mu \mathrm{m}, \gamma=-3.3, I=0.098)$, and the $\mathrm{LP}_{016}$ mode $(\Lambda=102 \mu \mathrm{m}, \gamma=-2.2, I=0.075)$, respectively. The value of $a_{1}$ for each grating is set to ensure complete rejection at the nominal resonance wavelength $1.55 \mu \mathrm{m}$. As shown in Fig. 6(b), for the gratings operating with the $\mathrm{LP}_{07}$ and $\mathrm{LP}_{09}$ modes $(\gamma>0)$, the resonance wavelength shifts to a longer wavelength from $1.55 \mu \mathrm{m}$, and the side lobes appear on the short-wavelength side of the rejection band, while for the grating operating with the $\mathrm{LP}_{014}$ and $\mathrm{LP}_{016}$ modes $(\gamma<0)$, the resonance wavelength shifts to a shorter wavelength from $1.55 \mu \mathrm{m}$, and the side lobes appear on the long-wavelength side of the rejection band. According to (6), the resonance wavelength shift increases with the product of $|\gamma|$ and $\Lambda$. For a uniform grating, the 3-dB bandwidth also increases with the product of $|\gamma|$ and $\Lambda$ [18], and this should also be true for an apodized grating. The grating for the $\mathrm{LP}_{09}$ mode has the largest value of $|\gamma| \Lambda$ and therefore exhibits the largest resonance wavelength shift and 3-dB bandwidth, as shown in Fig. 6(b). Note that the ac index changes for the four gratings are different. The grating operating with the cladding mode that has a larger overlap integral $I$ requires a smaller ac index change to achieve the same coupling coefficient - see (11), so the dc index

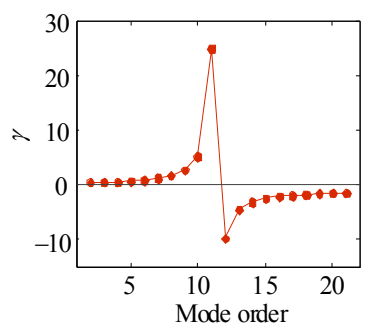

(a)

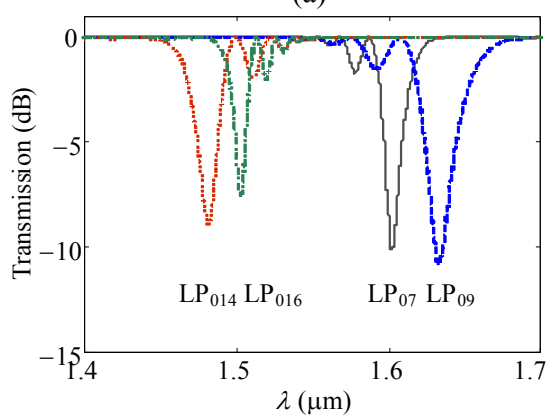

(b)

Fig. 6 Effects of the cladding-mode orders: (a) modal dispersion factor of the first twenty cladding modes at $1.55 \mu \mathrm{m}$ and (b) transmission spectra of four 60-mm-length raised-cosine apodized LPFGs with $a_{0} / a_{1}=1.0$, which are designed for operation with the $\mathrm{LP}_{07}, \mathrm{LP}_{09}, \mathrm{LP}_{014}$ and $\mathrm{LP}_{016}$ cladding modes, respectively. 
change used for comparison is smaller (since $a_{0} / a_{1}=$ 1.0 is fixed). This explains why the grating for the $\mathrm{LP}_{09}$ mode has a stronger rejection band and slightly weaker side lobes.

\subsection{Duty cycle of index modulation}

An LPFG is commonly written with a laser, which can be an ultra-violet (UV) laser $[1,8]$, a $\mathrm{CO}_{2}$ laser [19], or a femtosecond-pulse laser [20]. The index change induced along the fiber can be positive or negative, depending on the writing mechanism, but is usually of the same sign. In practice, it is difficult to eliminate the average index variation in a simple laser-written apodized LPFG.

Figure 7(a) shows the index profile of a practical laser-written apodized grating, where positive index changes are induced in areas defined by the narrow rectangular windows, which represent the openings along an amplitude mask or the irradiation spots of the writing laser beam in the point-by-point writing method. It is sufficient to consider the dc component and the fundamental spatial frequency of the profile, which can be obtained from the first two Fourier series coefficients of the rectangular function:

$$
\begin{gathered}
a_{0}=\frac{w}{\Lambda} \Delta n_{0} \\
a_{1}=\frac{2}{\pi} \sin \left(\frac{w}{\Lambda} \pi\right) \Delta n_{0}
\end{gathered}
$$

where $\Delta n_{0}$ is the maximum induced-index change, and $w$ is the width of the rectangular window. Figure 7(b) shows how the ratio $a_{0} / a_{1}$ decreases with $w / \Lambda$, the duty cycle of the index modulation. The result in Fig. 7(b) is general (i.e., independent of the actual grating profile). To minimize the dc component as much as possible, we should use the smallest possible duty cycle, which is limited by the maximum index change that can be induced in the fiber and the spot size of the writing laser beam. However, the smallest ratio of $a_{0} / a_{1}$ that can be achieved by decreasing the duty cycle is 0.5 , which makes complete elimination of side lobes impossible. Figure 7(c) shows the dependence of the contrast of the most significant side lobe on the grating profile for a grating with $a_{0} / a_{1}=0.5$ and $L=60 \mathrm{~mm}$, for three resonance contrasts: $10 \mathrm{~dB}, 20 \mathrm{~dB}$, and $30 \mathrm{~dB}$. The size of the side lobe increases with the contrast of the rejection band. The grating for the $\mathrm{LP}_{09}$ mode has a smaller side lobe than the grating for the $\mathrm{LP}_{07}$ mode because of the larger overlap integral of the $\mathrm{LP}_{09}$ mode. In general, one should choose a broader apodization profile and a cladding mode with a larger overlap integral $I$ in the design of apodized LPFGs. It should be noted that side lobes are present on both sides of the rejection band of a uniform grating. Apodization with dc index variation can at least remove the side lobes on one side, though it cannot suppress the side lobes on the other side.

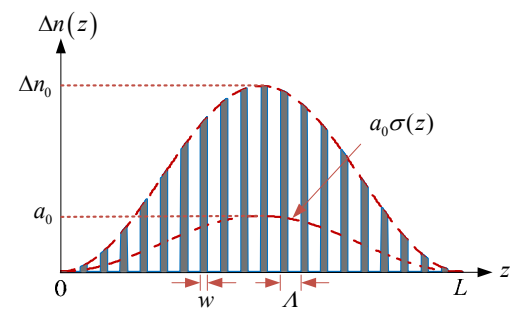

(a)

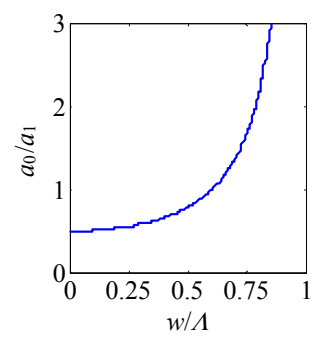

(b)

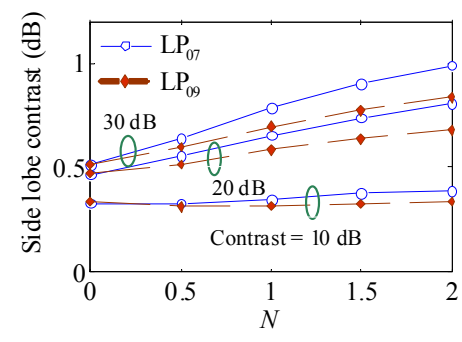

(c)
Fig. 7 Characteristics of a typical laser-written apodized LPFG: (a) index profile, (b) dependence of the ratio $a_{0} / a_{1}$ on the duty cycle $w / \Lambda$ of the index modulation, and (c) dependence of the contrast of the most significant side lobe on the apodization profile and the mode order, calculated for $a_{0} / a_{1}=0.5$ and the resonance contrasts $10 \mathrm{~dB}, 20 \mathrm{~dB}$, and $30 \mathrm{~dB}$.

To remove the side lobes on both sides, it is necessary to generate an apodized profile that has no dc index variation along the grating, as shown in Fig. 8. Such a profile can be produced either by post-processing of the grating shown in Fig.7(a) or 
by direct laser writing [21]. It is possible to vary the duty cycle of the index modulation along the grating to control the dc component. For example, two index profiles with complementary duty cycle distributions can be added together by double exposure to produce a uniform average index [8].

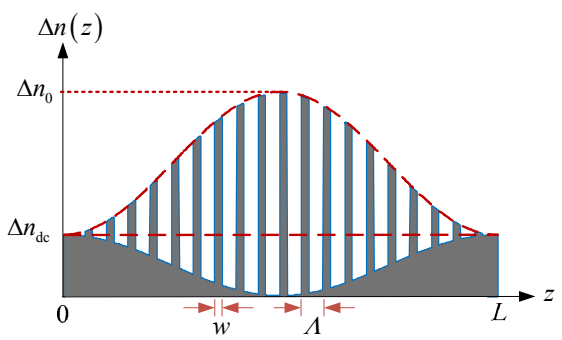

Fig. 8 Index profile of an apodized LPFG that has a uniform average index.

\section{Comparison with experimental results}

In this section, we analyse two laser-written apodized LPFGs, of which experimental results $[7,8]$ are available for comparison.

The first apodized grating is written with a $\mathrm{CO}_{2}$ laser, where the apodization function is a Gaussian function approximated piece-wisely with five uniform sections of equal lengths (10 periods each) [7]. The spot size of the laser beam is about $50 \mu \mathrm{m}$, and the grating period is $370 \mu \mathrm{m}$, so the duty cycle is about 0.14 . The grating is designed for coupling to the $\mathrm{LP}_{07}$ mode. In the simulation, the effective indices of the modes are adjusted slightly to fit the resonance wavelength of the experimental grating. The modal dispersion factor $\gamma$ is 1.1, and the overlap integral $I$ is 0.19 . Figure 9 compares the calculated spectrum $(M=5)$ with the measured spectrum. The calculated contrast of the side lobe on the short-wavelength side of the rejection band is $0.41 \mathrm{~dB}$, while that on the other side is $0.14 \mathrm{~dB}$ (the inset in Fig. 9). The agreement between the simulation and the experimental results is good. That side lobes are present on both sides is due to the use of a small number of sections. A grating with the same length and period, written without purposely introducing apodization, shows a side lobe of $0.51 \mathrm{~dB}$ on one side and $0.66 \mathrm{~dB}$ on the other side of the rejection band [7].

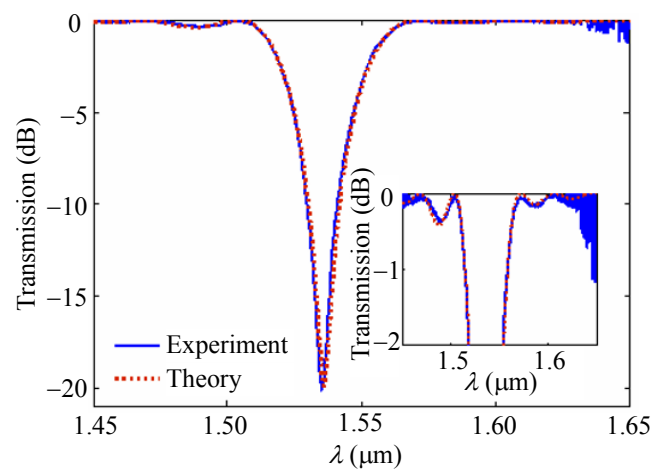

Fig. 9 Measured (solid) [7] and calculated (dotted) transmission spectra of a piece-wise Gaussian-apodized LPFG written with a duty cycle of $w / \Lambda=0.14$.

The second grating for comparison is a UV-laser written grating that has a constant index change and a gradually varying duty cycle of index modulation along the grating [8]. The grating has a period of $395 \mu \mathrm{m}$ and a length of $50 \mathrm{~mm}$, and is designed for coupling to the $\mathrm{LP}_{06}$ mode. In the simulation, the effective indices of the modes are chosen to fit the resonance wavelength of the experimental grating. The modal dispersion factor $\gamma$ is 0.9 , and the overlap integral $I$ is 0.1 . Figure 10 compares the calculated spectrum (using $M=15$ ) with the measured spectrum, where the inset shows the variation of the duty cycle along the grating. This grating consists of a large $\mathrm{dc}$ index variation and therefore exhibits very strong side lobes (up to $9.5 \mathrm{~dB}$ ) on the short-wavelength side of the rejection band. The

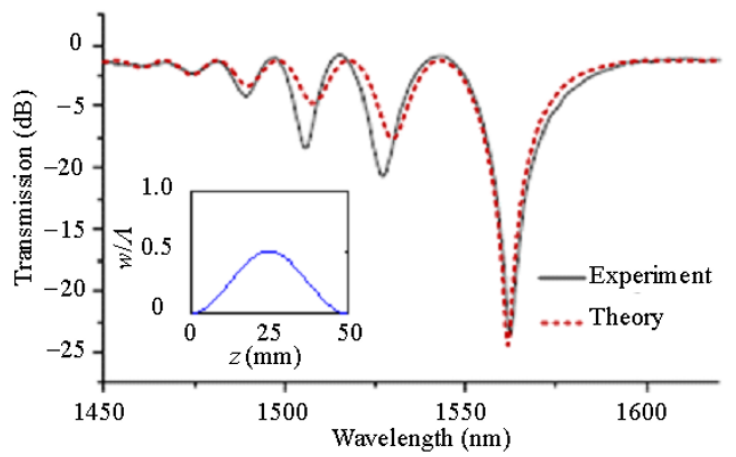

Fig. 10 Measured (solid) [8] and calculated (dotted) transmission spectra of an LPFG that has a varying duty cycle along the grating (inset). 
agreement between our simulation and the experimental results is reasonable, considering the fact that many of the fiber parameters of the experimental grating are not known.

\section{Conclusions}

We have applied the transfer matrix method to the analysis of the effects of average index variation on the transmission spectrum of an apodized LPFG. We have studied in detail how these effects depend on the grating length, the grating profile shape, the modal dispersion factor, and the duty cycle of the index modulation. We find that, for a fixed ac index modulation, the dc index variation can shift the resonance wavelength and at the same time, weaken and broaden the rejection band. While the idea of using apodization is to suppress side lobes, our analysis indicates that the presence of the average index variation in an apodized LPFG makes complete suppression of side lobes impossible. In fact, the side lobes generated by a sufficiently large average index variation can be much stronger than those of a uniform grating. The generated side lobes appear mainly on one side of the rejection band, which can be the short-wavelength or long-wavelength side, depending on the signs of the dc index change and the modal dispersion factor. To reduce the side lobes as much as possible in laser writing of apodized LPFGs, we should use the smallest possible duty cycle of the index modulation together with a apodization profile that varies slowly towards the two ends of the grating. Compared with apodized FBGs [9, 11], apodized LPFGs are much more tolerant against the average index variation, which can ease the fabrication process for side-lobe suppression, as verified by the $\mathrm{CO}_{2}$-laser written apodized LPFGs demonstrated recently [7].

\section{Acknowledgment}

The authors thank Dr. Qing Liu for useful discussions. This research work was supported by a grant from CityU, under Project 7002444.
Open Access: This article is distributed under the terms of the Creative Commons Attribution License which permits any use, distribution, and reproduction in any medium, provided the original author(s) and source are credited.

\section{References}

[1] A. M. Vengsarkar, P. J. Lemaire, J. B. Judkins, V. Bhatia, T. Erdogan, and J. E. Sipe, "Long-period fiber gratings as band-rejection filters," Journal of Lightwave Technology, vol. 14, no. 1, pp. 58-65, 1996.

[2] K. S. Chiang and Q. Liu, "Long-period grating devices for application in optical communication," in Proceedings of 5th International Conference on Optical Communications and Networks and 2nd International Symposium on Advances and Trends in Fiber Optics Applications (ICOCN/ATFO), Chengdu, China, September, pp. 128-133, 2006.

[3] R. Slavík, Y. Park, M. Kulishov, R. Morandotti, and J. Azaña, "Ultrafast all-optical differentiators," Optics Express, vol. 14, no. 22, pp. 10699-10707, 2006.

[4] S. W. James and R. P. Tatam, "Optical fibre long-period grating sensors: characteristics and application," Measurement Science \& Technology, vol. 14, no. 5, pp. R49-R61, 2003.

[5] D. Nodop, C. Jauregui, F. Jansen, J. Limpert, and A. Tünnermann, "Suppression of stimulated Raman scattering employing long period gratings in double-clad fiber amplifiers," Optics Letters, vol. 35, no. 17, pp. 2982-2984, 2010.

[6] F. Y. M. Chan and K. S. Chiang, "Analysis of apodized phase-shifted long-period fiber gratings," Optics Communications, vol. 244, no. 1-6, pp. 233-243, 2005.

[7] Y. Gu, K. S. Chiang, and Y. J. Rao, "Writing of apodized phase-shifted long-period fiber gratings with a computer-controlled $\mathrm{CO}_{2}$ laser," IEEE Photonics Technology Letters, vol. 21, no. 10, pp. 657-659, 2009.

[8] X. Shu, K. Sugden, and I. Bennion, "Apodisation of photo-induced waveguide gratings using double-exposure with complementary duty cycles," Optics Express, vol. 16, no. 3, pp. 2221-2225, 2008.

[9] V. Mizrahi and J. E. Sipe, "Optical properties of photosensitive fiber phase gratings," Journal of Lightwave Technology, vol. 11, no. 10, pp. 1513-1517, 1993.

[10] H. Kogelnik, "Filter response of nonuniform almost-periodic structures," The Bell System Technical Journal, vol. 55, no. 1, pp. 109-126, 1976.

[11] J. E. Sipe, L. Poladian, and C. M. De Sterke, 
"Propagation through nonuniform grating structures," Journal of the Optical Society of America A: Optic-s and Image Science, vol. 11, no. 4, pp. 1307-1320, 1994.

[12] A. W. Snyder and J. D. Love. Optical Waveguide Theory. London: Chapman and Hall, 1983.

[13] T. W. MacDougall, S. Pilevar, C. W. Haggans, and M. A. Jackson, "Generalized expression for the growth of long period gratings," IEEE Photonics Technology Letters, vol. 10, no. 10, pp. 1449-1451, 2002.

[14] M. Yamada and K. Sakuda, "Analysis of almost-periodic distributed feedback slab waveguides via a fundamental matrix approach," Applied Optics, vol. 26, no. 16, pp. 3474-3478, 1987.

[15] H. Ke, K. S. Chiang, and J. H. Peng, "Analysis of phase-shifted long-period fiber gratings," IEEE Photonics Technology Letters, vol. 10, no. 11, pp. 1596-1598, 1998.

[16] M. Monerie, "Propagation in doubly clad single-mode fibers," IEEE Journal of Quantum Electronics, vol. 18, no. 4, pp. 535-542, 1982.
[17] T. Erdogan, "Fiber grating spectra," Journal of Lightwave Technology, vol. 15, no. 8, pp. 1277-1294, 1997.

[18] X. Shu, L. Zhang, and I. Bennion, "Sensitivity characteristics of long-period fiber gratings," Journal of Lightwave Technology, vol. 20, no. 2, pp. 255-266, 2002.

[19] D. D. Davis, T. K. Gaylord, E. N. Glytsis, S. G. Kosinski, S. C. Mettler, and A. M. Vengsarkar, "Long-period fibre grating fabrication with focused $\mathrm{CO}_{2}$ laser beams," Electronics Letters, vol. 34, no. 3, pp. 302-303, 1998.

[20] Y. Kondo, K. Nouchi, T. Mitsuyu, M. Watanabe, P. G. Kazansky, and K. Hirao, "Fabrication of long-period fiber gratings by focused irradiation of infrared femtosecond laser pulses," Optics Letters, vol. 24, no. 10, pp. 646-648, 1999.

[21] V. Grubsky, A. Skorucak, D. S. Starodubov, and J. Feinberg, "Fabrication of spectrally-clean, long-period grating filters," in Technical Digest of Optical Fiber Communication Conference, San Diego, Feb. 21-26, pp. 174-176, 1999. 